\title{
Fardeau des maladies chroniques en Afrique subsaharienne : plaidoyer pour une mise en place des soins palliatifs et d'accompagnement en fin de vie en République démocratique du Congo
}

\section{Burden of chronical disease in sub-sharian Africa: A plea for implementation of palliative care and end of life support in Democratic Republic of Congo}

\section{J.M. Lofandjola ${ }^{a, b, *}$, E. Kiswaya Sumailic, J. Petermans ${ }^{d}$}
a Institut supérieur des techniques médicales de Kinshasa, République populaire du Congo
${ }^{\mathrm{b}}$ Faculté de médecine, département des sciences de santé publique, université de Liège, Belgique
c Faculté de médecine, université de Kinshasa, unité de néphrologie, République populaire du Congo
' Faculté de médecine, département de gériatrie, université de Liège, Belgique

Reçu le 23 mai 2017 ; accepté le 19 juin 2017

Disponible sur Internet le 18 juillet 2017

\section{MOTS CLÉS}

Afrique subsaharienne ;

Ampleur ;

Maladies chroniques ;

Soins palliatifs

\begin{abstract}
Résumé Le fardeau de maladies chroniques (surtout non transmissibles) est le défi majeur mondial de santé publique. La combinaison d'un développement économique erratique, des systèmes de santé inadaptés, manquant de capacités de diagnostic et thérapeutique, provoque dans beaucoup de pays à ressources limitées dont la République démocratique du Congo (RDC) une impossibilité à gérer l'ampleur actuelle de ces maladies. À cela s'ajoute la pauvreté répandue et les défis environnementaux. Dans ce contexte, le besoin des services de soins palliatifs (SP) pour des patients en fin de vie est énorme et indéniable. La formation et l'information des décideurs politiques, des soignants et familles sont nécessaires dans cette transition sanitaire. Les SP pourraient être la solution d'un accompagnement avec dignité, améliorant la qualité de
\end{abstract}

\footnotetext{
* Auteur correspondant. Faculté de médecine, école de santé publique, université de Liège, Sart-Tilman B23, B-4000 Liège, Belgique. Adresse e-mail : jlofandjola@student.ulg.ac.be (J.M. Lofandjola).
} 


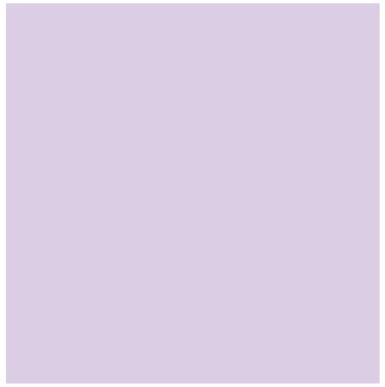

vie des malades chroniques. Cet article présente et analyse l'ampleur de ces maladies chroniques et cherche à faire un plaidoyer pour implémenter ces soins dans les pratiques médicales à Kinshasa. Trois raisons principales (transition épidémiologique, manque de formation de soignants des approches palliatives et de priorité par les décideurs politiques de ces maladies chroniques) devraient motiver tous les acteurs (partenaires, politiques, soignants et familles) à implémenter ces soins dans les pratiques médicales et de faire que ces maladies ne soient pas négligées en Afrique subsaharienne.

(c) 2017 Elsevier Masson SAS. Tous droits réservés.

\section{KEYWORDS}

Sub-saharan Africa;

Extent;

Chronic diseases;

Palliative care
Summary The burden of chronic diseases (especially non-communicable) is the world's major public health challenge. The combination of erratic economic development, inadequate health systems, lacking diagnostic and therapeutic capabilities, is provoking in many resource-limited countries including the Democratic Republic of Congo (DRC) an inability to manage the current extent of these diseases. Added to this is the widespread poverty and environmental challenges. In this context, the need for palliative care services for terminally ill patients is enormous and undeniable. Training and information for policy makers, caregivers and families are needed in the health transition. The palliative care could be the solution to a support with dignity, improving chronic patients' quality of life. This article presents and analyzes the extent of these chronic diseases and seeks to make a place to implement this care in medical practice in Kinshasa. Three main reasons (epidemiological transition, lack of training of caregivers of palliative approaches and priority by policy makers of these chronic diseases) should motivate all stakeholders (partners, political, caregivers and families) to implement these treatments in medical practices and to these diseases are not neglected in Sub-saharan Africa.

(c) 2017 Elsevier Masson SAS. All rights reserved.

\section{Introduction}

Les maladies chroniques (MC), à ce jour, un fardeau à l'échelle mondiale, mettent les malades dans un besoin accru et prolongé de soutien médical [1]. Les MC sont les affections chroniques de longue durée (plus de 6 mois) qui évoluent et persistent dans le temps. Elles nécessitent des soins de santé depuis plus de 6 mois (maladies cardiovasculaires, maladies oncologiques, maladies respiratoires chroniques, diabète et le $\mathrm{VIH} /$ sida), sont responsables de $63 \%$ des décès [2]. Relativement peu d'informations fiables concernant ces maladies sont disponibles. Des listes reprenant ces principales maladies chroniques existent mais elles sont évolutives et ne font pas toujours l'unanimité [1].

Aujourd'hui, il y a cette volonté et tendance d'augmenter l'espérance de vie, raison majeure à développer la prise en charge des maladies chroniques.

L'Afrique connait une augmentation de l'incidence des MC avancées nécessitant une prise en charge palliative précoce. La démarche palliative intégrée encore appelée SP intégrés, permettent d'accompagner le patient, d'adapter la prise en charge soignante, thérapeutique, sociale, humaine tout au long de la maladie avec des visées qui peuvent être en partie curative et en partie palliative ou les deux à la fois. C'est au fond une " assurance » de continuité de la prise en charge humaine et sociale. Ceuxci sont une des composantes de soins globaux dans la prise en charge des maladies chroniques. Les services des SP ne sont pas opérationnels dans la plupart des pays de l'Afrique sub-saharienne. Seulement six pays le Kenya, le Rwanda, l'Afrique du Sud, le Swaziland, la Tanzanie et l'Ouganda les ont intégré à leurs plans de santé nationaux [3]. De ces pays, le Kenya, le Malawi, l'Afrique du Sud et l'Ouganda reconnaissent les SP comme une matière à enseigner dans les écoles de médecine et dans le programme d'études des professionnels de santé [3]. La formation en SP devrait se faire de manière continue. Toute personne intéressée, décideurs, soignant et famille est appelée à bénéficier de l'éducation et information relatives aux aspects des SP.

Dans la plupart des pays subsahariens, les MC et évolutives ne sont pas encore considérées comme prioritaires [4]. Et pourtant, elles ont actuellement tendance à augmenter dans cet environnement où sévit les pathologies infectieuses. Les MC constituent une menace pour la santé publique et pour les budgets de santé à travers le monde [5]. Cette question de l'allocation des ressources surtout dans les zones où elles sont limitées semble être problématique. Les décès dus aux maladies infectieuses baisseront de $3 \%$ durant la prochaine décade alors que ceux liés aux MC augmenteront de $17 \%$ [6]. Cette augmentation concernera essentiellement les pays à revenu faible dont ceux d'Afrique subsaharienne (ASS) [7-10]. Sur les 36 millions de personnes décédées de maladies chroniques en 2008, $29 \%$ avaient moins de 60 ans et la moitié étaient des femmes [11]. Ces MC, notamment le VIH/SIDA et certaines maladies non transmissibles (MNT) sont les causes actuelles 
de multiples hospitalisations dans l'ASS. Le soutien médical doit être pluri-disciplinaire. À Kinshasa, un adulte sur quatre serait à haut risque des MNT [12]. Le changement de mode de vie (tabagisme, alimentation déséquilibrée, usage nocif de l'alcool, sédentarité « inactivité », croissance d'urbanisation, recours aux moyens de transport motorisés) dans la plupart des pays à faible revenu en serait responsable [12].

Dans certains pays d'ASS, la prise en charge du diabète consomment déjà $15 \%$ du budget des soins de santé nationaux [5]. Dans les zones rurales, plus de $80 \%$ des personnes sont diagnostiqués à des stades avancés de leurs pathologies virales [13].

Malgré ce constat, aucune transition vers les SP ne semble se développer dans l'organisation des soins de santé dans les pays d'Afrique francophone où l'on note que 11 pays sur dix-huit dont la RDC ne pratiquent aucune forme de soins palliatifs [3].

Et pourtant, la seule réponse globale envers cette crise sanitaire et socioéconomique serait la mise en place progressive de ce type de soins. Cela améliora la qualité de vie des malades chroniques en fin de vie et des familles par un soutien médical contextualisé $[14,15]$. Les soins palliatifs sont des soins médicaux qui améliorent la qualité de vie physique, sociale, psychologique et spirituelle du patient et de sa famille et vont au-delà de soulager la douleur. Ils correspondent aux soins actifs et complets administrés aux patients dont l'affection (maladie) ne répond plus au traitement curatif.

Cet article présente et analyse le fardeau des maladies chroniques et cherche à faire un plaidoyer pour implémenter ces soins dans les pratiques médicales à Kinshasa et en RDC. Trois raisons principales (transition épidémiologique, manque de formation de soignants des approches palliatives et de priorité par les décideurs politiques de ces maladies chroniques) devraient motiver tous les acteurs (partenaires, politiques, soignants et familles) à implémenter ces soins dans les pratiques médicales et faire en sorte que ces maladies ne soient plus négligées en Afrique subsaharienne.

\section{État de lieux des maladies chroniques en Afrique subsaharienne (ASS)}

La majorité des pays d'Afrique connaissent un double défi, celui des maladies infectieuses aigues et des maladies chroniques $[16,17]$. Plus de $80 \%$ d'adultes de plus de 60 ans ont au moins une affection chronique [1]. Les modèles de multi-morbidité des malades des pays africains diffèrent de ceux des pays développés par le fait de l'accessibilité géographique et financière des soins [17]. Par exemple en comparaison avec certains pays à haut revenu, plusieurs travaux montrent que les prévalences de maladies rénales chroniques (MRC) atteignent ou dépassent dix pourcent dans les pays riches tels qu'États-Unis [18], Espagne [19], Japon [20], Canada [21], Italie [22] et Norvège [23]. Comme dans les pays riches, cette prévalence de MRC à Kinshasa est de 12,4\% [24]. Dans les pays africains, plus des 3,7 millions de décès causés par les MNT ont été enregistrés en ASS en 2008, mais si aucune action n'est entreprise, elles atteindront les 14 millions d'ici 2050 [25].
Les maladies cardio-vasculaires (MCV) sont responsables du plus grand nombre de décès dus aux MNT (17,3 millions de décès par an), suivies des cancers ( 7,6 millions), des maladies respiratoires $(4,2$ millions) et du diabète $(1,3$ millions) [26]. Les données de la littérature montrent qu'en 2012, environ 35,3 millions de personnes vivaient avec le VIH dans le monde. La prévalence de diabète en 2014 était estimée à $9 \%$ chez les adultes âgés de 18 ans et plus. Il est prévu que cette prévalence augmente de $80 \%$ au cours des 20 prochaines années [27]. En 2012, on comptait au moins 14 millions de nouveaux cas de cancers [28]. Plus de $60 \%$ des nouveaux cancers surviennent en Afrique et autres pays à revenu faible. Ces régions représentent $70 \%$ des décès par cancer dans le monde [28]. Par manque des moyens d'investigations appropriées et la résilience économique, le diagnostic se fait souvent à un stade avancé de maladie, souvent irréversible. Le manque de soutien financier retarde le développement des capacités de prévention, le traitement dans son ensemble et la recherche dans la plupart de ces pays. Parfois pour les pathologies tumorales les moyens disponibles de chimiothérapie/radiothérapie existent, mais les patients n'accèdent pas par manque des moyens financiers. Malgré des signes croissants d'impact épidémiologique et économique de ces $M C$, la réponse globale au problème reste insuffisante et inadaptée eu égard aux besoins. Les décideurs n'ont pas de données réelles fournies par les « intervenants du terrain ». Les préjugés que ces maladies touchent seulement les riches et les personnes âgées persistent largement [29].

En République démocratique du Congo (RDC), près d'un adulte sur quatre serait à haut risque des MNT [12]. En effet, la prévalence de l'hypertension (HTA) tant en milieu rural qu'urbain se situe entre 25-30\% [16]. Selon Katchunga [30] à l'Est de la RDC, 57,5 \% des hypertendus n'étaient au courant de leurs maladies et seulement 13,6\% avaient un suivi médical. Dans une autre zone de santé semi-rurale de la RDC, la protéinurie (micro-albuminurie et macro-albuminurie) était fréquemment détectée pendant le dépistage du diabète, de l'obésité et l'hypertension et était particulièrement associée aux patients de plus de 60 ans. Or, la gestion intégrée du diabète est essentielle pour prévenir l'insuffisance rénale dans cette population [31]. Plusieurs auteurs ont confirmé que l'hypertension artérielle, le diabète, l'âge, le VIH et l'obésité sont des facteurs déterminants des maladies cardiovasculaires et de l'insuffisance rénale à Kinshasa [16,24,32,33]. D'autres études ont montré une fréquence de la rétinopathie diabétique qui augmente avec l'âge du patient et la durée du diabète [34,35]. En ASS, plusieurs maladies tropicales (filariose, schistosoma mansoni, la trypanosomiase humaine africaine, la malaria avec plasmodium malariae, la lèpre et la tuberculose encore répandue en Afrique peuvent aussi affecter les reins [36]. La prévalence du VIH était de 4,3\% [12] en 2008 et de $3,2 \%$ en 2009 [37] pour les personnes de tranche d'âge de 15-49 ans, de 1,2\% dans la population générale en 2013[38] et de $4,6 \%$ [39] en milieu rural en RDC. Les résultats de l'EDS-RDC II 2013-2014 situent actuellement la prévalence du VIH en RDC à 1,2\% avec des différences entre les provinces. Que ce soit chez les femmes ou les hommes, c'est au Maniema (respectivement, 3,9\% et $4 \%$ ) que les niveaux de prévalence sont les plus élevés, suivi de la province Orientale (respectivement $3,9 \%$ et $0,5 \%$ ), de Kinshasa 
(respectivement $2,6 \%$ et $0,3 \%$ ) et du Kasaï Oriental (respectivement $2,4 \%$ et $1,1 \%$ ). Au nord-Est de la RDC, $24 \%$ souffraient de cancer de la peau [40]. À Kinshasa, Budiango et al. [41] ont trouvé que $66,7 \%$ avaient un diagnostic de lymphome de Burkitt et que la majorité des patients $(82,5 \%)$ avaient un stade avancé (étape III et IV) auprès des enfants âgés d'au moins 8 ans. À Lubumbashi, l'unité d'oncologie pédiatrique et le centre ophtalmologique des cliniques universitaires ont lancé depuis le début de 2013 un programme de sensibilisation des masses sur le rétinoblastome avec l'appui de plusieurs organismes dont l'Organisation mondiale du cancer [42].

Selon l'Organisation mondiale de la santé (OMS), bien que les moyens et les connaissances pour soulager la douleur existent, la douleur des enfants n'est souvent pas reconnue, elle est ignorée, voire niée [43].

La formation à l'évaluation de la douleur chez les enfants est particulièrement importante étant donné que les enfants plus jeunes peuvent ne pas être en mesure d'exprimer si oui ou non ils souffrent. La stratégie de SP devrait s'intéresser aussi des enfants atteints des pathologies chroniques. La création des unités spécialisées des soins pédiatriques pourrait garantir des soins appropriés adaptés à l'âge pour les enfants ayant une douleur chronique de longue durée. Inclure la morphine sous forme sirop et liquide dans la liste des médicaments essentiels est essentiel pour répondre efficacement à une douleur chronique de l'enfant.

\section{Politiques actuelles de lutte contre les maladies chroniques}

Les systèmes de santé des pays d'ASS sont prioritairement orientés pour combattre les maladies aigues telles que les maladies infectieuses et parasitaires au détriment des MC [44]. La plupart des pays en ASS appliquent les soins de santé primaires (SSP) comme stratégies essentielles. En effet la stratégie ciblant en priorité les soins primaires mise en place fin des années 70 devrait évoluer car les besoins ont changé. Dans ces SSP, les soins curatifs et de réadaptation, préventifs et promotionnels sont les éléments de base. Ces systèmes de soins et la formation des soignants restent focalisés à ces stratégies des soins essentiels et intégrés. Avec l'escalade des MC, l'adaptation de ce type de soins vers les SP ne semblent pas encore appliquée. Des innovations des systèmes des soins orientés vers les nouvelles approches types soins continus des MC sont attendus [45].

En pratique, les hôpitaux connaissent une croissance importante des MC sans moyens de prise en charge adaptée. Moins de 0,1\% de 2,9 milliards de dollars en 2002 a été consacré aux MC en Afrique [46]. Plus de la moitié du budget de santé des pays africains provient de l'extérieur. De cette aide étrangère, moins de $3 \%$ est consacrée aux MNT [47].

Dans ce contexte, l'éducation du patient et de sa famille est un élément clé de la gestion des MC. Dans l'accompagnement de ces malades, il est nécessaire de tenir compte d'un facteur important, la douleur, qui influence considérablement la qualité de vie pendant plusieurs années. Pour cela la mise en place progressive et l'application des SP s'avèrent nécessaires. Les politiques et les planificateurs de santé devraient être sensibilisés pour un changement des pratiques médicales. Dans la prise en charge des douleurs chroniques, la morphine est parfois le seul et le médicament de choix le plus utilisé [48]. Des réglementations trop restrictives pour la morphine et d'autres médicaments essentiels sous contrôle empêchent l'accès à un soulagement suffisant de la douleur et aux soins palliatifs [49]. Cela pourrait entrainer une mauvaise gestion de la douleur auprès des patients [50].

En RDC, à l'instar d'autres pays d'ASS, la problématique des soins des malades chroniques reste évidente. De nombreux programmes de ministère de santé interviennent dans la prise à charge de ces maladies. Ces programmes existent au ministère de la santé, mais ne sont pas opérationnels, manquant les données réelles de terrain. Il est temps d'intégrer les aspects des SP dans ces programmes existants contre les maladies cardio-vasculaires, le diabète et le VIH/SIDA. Le renforcement des campagnes de sensibilisation sur le dépistage et la prévention de maladies chronique semble utile afin de limiter l'impact de ces maladies. Le système d'enregistrement des données MNT reste inopérable et les données restent parcellaires. Cela résulte probablement d'un manque de coordination efficace et efficiente entre le pouvoir public et les organisations non gouvernementales qui s'occupent, accueillent et prennent en partie des soins de santé de la population.

Le ministère de santé de la RDC devra encourager la mise en place de SP dans les soins à travers ses ressources. Agir dans un contexte de faible ressources est un dilemme éthique pour les soignants, afin d'assurer entre autres l'autonomie, la bienfaisance et la juste allocation de ressources limitées envers les malades chroniques en fin de vie [51]. À ce jour, le système est en train de se relancer par la réforme du secteur de santé (récemment, en RDC, le ministère de la santé publique a reconnu que les SP n'étaient pas pris en compte dans le Programme national de développement sanitaire 2011-2015 et aujourd'hui tout était mis en marche pour intégrer cette nouvelle approche dans le système de santé congolais. Il y a eu l'organisation des premiers et deuxième congrès internationaux des soins palliatifs (2013 et 2015), le colloque des soins palliatifs pédiatriques (2015) et la création d'une bibliothèque virtuelle spécialisée en soins palliatifs en 2016), mais la situation n'est pas encore optimale.

\section{Plaidoyer pour un changement de politique des soins basés sur l'amélioration et les soins palliatifs}

Les estimations montrent que $60 \%$ au moins de la population dans cette partie du monde habiteraient les villes d'ici 2030 [52]. L'exode rural oblige un grand nombre des personnes à s'installer dans les périphéries des villes. Cela fait suite au conflit armé de plus de vingt ans qui sévit à l'est du pays. Cette population fréquente moins les services de santé lors d'épisode de maladie. Elle consulte facilement les guérisseurs traditionnels, qui leurs proposent des approches holistiques et culturelles acceptables. Certaines études ont montré que lors d'épisode de maladie, le recours à un tradipraticien est de $21 \%[53,54]$. 
Plusieurs familles croient aux guérisons miracles et ne tolèrent pas l'administration de morphine prétextant que cette molécule pousse calmement le patient gravement malade vers la mort [51].

Les systèmes de santé sont faibles. Les besoins en santé toujours élevés et non proportionnels aux ressources humaines et matérielles. Une étude récente [55] a montré qu'à Kinshasa il y a un médecin spécialiste pour plus de 59000 habitants en chirurgie, en gynécologieobstétrique, en médecine interne, en pédiatrie et en anesthésie-réanimation. Dix hôpitaux de la capitale estimés couvrir la quasi-totalité de la ville-province de Kinshasa totalisent ainsi 54 chirurgiens, 39 gynécologues, 35 internistes et 16 pédiatres, un effectif en fait de loin inférieur à la réalité vue la difficulté de répertorier tous ceux qui travaillent dans d'innombrables structures privées. D'un autre côté, les médecins RDC ont une polyvalence plus marquée ce qui peut être une chance pour l'implantation d'unité de SP.

Cette épidémie de $21^{\mathrm{e}}$ siècle que sont les $M C$ a un impact en termes de coûts financiers, humains et surtout organisationnels. Ces maladies restent évitables, si les politiques publiques s'attaquent aux facteurs communs (la consommation de tabac, d'alcool, les régimes alimentaires néfastes pour la santé et la sédentarité). Ces pays devront promouvoir la prestation de soins de santé universels s'appuyant sur la sensibilisation, l'éducation et la formation des pratiques palliatives en fin de vie.

Toutefois le plus souvent, ce sont les organisations non-gouvernementales et confessionnelles qui se chargent actuellement de ces soins dans cette partie du monde [3]. Les malades chroniques sont prises en charge dans les unités de soins traditionnelles avec les personnels moins spécialisés dans cette approche palliative.

Humaniser la fin de vie du patient, soulager la douleur, apaiser la souffrance physique, sauvegarder la dignité de la personne malade et soutenir ses proches justifient la mise en place des SP. De nos jours, l'Organisation mondiale de santé (OMS) souhaite que les SP soient intégrés comme une composante des soins dans les politiques de santé, surtout que la majorité de la population n'a pas accès à ces soins dans les pays à faible revenu [49].

En RDC, il y a à peine 2000 médecins pour 70 millions d'habitants [16]. Par contre en Belgique, pour 11 millions d'habitants, il y a plus de 56000 médecins [56]. Le ratio médecin/habitant rend en partie difficile la mise en application des SP en RDC.

Mettre en place les formations continues des soignants des zones de santé (axe stratégique de base) et introduire la formation basique des SP dans le curriculum des soignants rendra ces soins faisables dans les pratiques médicales à Kinshasa et à l'intérieur du pays. Ces soins auront des effets bénéfiques sur les malades en fin de vie et les familles s'ils sont intégrés dans les programmes existants. La notion de famille en Afrique englobe la famille biologique, les amis et les connaissances de longue date. Le concept africain de famille est fondé sur la solidarité fraternelle. Lors des SP, le soignant, la famille et le malade forment un tout. Cette relation doit rester professionnelle, objective et intentionnelle. Certains médecins à Kinshasa disent tout simplement au malade en état palliatif de retourner à domicile. Par manque des moyens financiers, les personnes préfèrent mourir à domicile [57-59]. Devant ces maladies sans solution de prise en charge appropriée dans nos systèmes de santé, les soins palliatifs sont à encourager au sein de la communauté pour cette réalité et problème de santé publique. Le soulagement de la souffrance au moyen d'une identification précoce, de la douleur et d'autres problèmes physiques, psychosociaux et spirituels aidera le malade en fin de vie.

En RDC, comme dans d'autres pays d'Afrique, le secteur de santé n'est pas épargné par l'effondrement de l'économie erratique, conséquence des conflits armés et surtout de la mauvaise gestion des ressources. Cela a pour conséquence les ruptures fréquentes entre le niveau central et les points d'utilisation dans la chaine d'approvisionnement en médicaments [60]. Le système d'approvisionnement des médicaments essentiels est complétement déréglé en RDC ; même concernant la morphine, molécule la plus utilisée pour soulager les douleurs chroniques [61]. Ce pays (la RDC) compte 21 villes [7]. L'approvisionnement de l'ensemble des médicaments essentiels se fait surtout dans les grandes villes et à Kinshasa. Les malades chroniques des zones rurales (où vivent $70 \%$ de la population) requérant des soins palliatifs semblent être abandonnés, sans aide médicale spécifique ni soutien en matière de douleurs physiques, morales, sociales et psychologiques. La mise en place de SP serait la solution d'un accompagnement avec dignité, améliorant la qualité de vie des malades chroniques.

\section{Conclusion}

Cet article présente et analyse le fardeau des MC et le manque de SP dans les pratiques médicales en ASS. La mise en pratique de ces approches palliatives pour ces patients souffre d'un manque de ressources, de connaissances et de preuves. D'où l'intérêt d'implémenter ces soins dans les pratiques médicales pourrait améliorer la qualité de vie de ces patients et de leurs familles. Les pratiques médicales méritent d'être réexaminées pour intégrer ces approches. La formation et l'information des décideurs politiques, des soignants et familles sont nécessaires dans cette transition sanitaire. La première étape pour implémenter ces SP devra se faire par la formation et l'information de soignants (médecins, infirmiers et toute personne intéressée) dans les zones de santé, dans les écoles et les autres milieux de soins. La deuxième serait de sensibiliser les responsables de secteur sanitaire, les décideurs politiques et le grand public (familles et toute personne intéressée). La troisième serait de revisiter les référentiels de formation en SP (existant ailleurs) pour les adapter à la réalité de l'ASS.

Nous défendons l'implémentation de ces approches dans les soins pour des raisons d'intérêt scientifique d'avenir et surtout pour améliorer la qualité de vie du patient et de sa famille pour un accompagnement humanisé.

\section{Remerciements}

Nous remercions tous les co-auteurs qui ont contribué à la conduite de cette réflexion et à la rédaction du manuscrit.

Nos remerciements s'adressent également au Professeur Amuli Jiwe Jean Pierre de l'Institut supérieur des techniques 
médicales de Kinshasa pour le soutien pendant la réalisation de l'article.

\section{Déclaration de liens d'intérêts}

Les auteurs déclarent ne pas avoir de liens d'intérêts.

\section{Références}

[1] Sadhna D, Christ G [Council on social work and education. Consulté le 16/02/2015] Chronic illness and aging: the demographics of aging and chronic diseases; 2003 http: / / www. cswe.org/File. aspx?id=25462.

[2] Organisation mondiale de la santé. Maladies chroniques; 2013 [Consulté le 16/02/2015] http://www.who.int/ topics/chronic_diseases/fr/.

[3] Mwangi-Powell F, Dix O. Palliative care in Africa: an overview. Africa Health 2011 [Consulté le 16/02/2015] http:// Africahealth. com/articles/july_2011/P_care_overview.pdf.

[4] Uwakwe R, Ibeh CC, Modebe Al, Bo E, Ezeama N, Njelita I, et al. The epidemiology of dependence in older people in Nigeria: prevalence, determinants, informal care, and health service utilization. A 10/66 dementia research group cross-sectional survey. J Am Geriatr Soc 2009;57:1620-7.

[5] Chan M. Obesity: bad trouble is on its way; 2012 [Consulté le 16/02/2015] http://www.who.int/dg/ speeches/2012/forum_20120921/en/.

[6] Fondation du rein. Journée mondiale Rein; 2006 [Consulté le 17/09/2014] http://www.nephromaroc.org.

[7] Ministère du plan RDC. Deuxième enquête démographique et de santé (EDS-RDC II 2013-2014): rapport préliminaire sur la prévalence du VIH; 2014. p. 10 [http://pdf.usaid.gov/pdf_docs/pbaaa437.pdf].

[8] Boutayeb A. The double burden of communicable and noncommunicable diseases in developing countries. Trans R Soc Trop Med Hyg 2006;100:191-9.

[9] Boutayeb A, Boutayeb S. The burden of non communicable diseases in developing countries. Int J Equity Health 2005;4:2.

[10] Harding R, Gwyther L, Mwangi-Powell F, Powell RA, Dinat N. How can we improve palliative care patient outcomes in lowand middle-income countries? Successful outcomes research in sub-saharan Africa. J Pain Symptom Manage 2010;40:23-6.

[11] Organisation mondiale de la santé. Les maladies non transmissibles; 2013 [http://www.who.int/mediacentre/ factsheets/fs355/fr/ consulté le 16/02/2015].

[12] Ministère du plan R.D.C. Enquête démographique et de santé (EDS-RDC); 2008. p. 220 [http://www.dhsprogram.com/ pubs/pdf/FR208/FR208.pdf].

[13] Kautako-Kiambi M, Aloni-Ntetani M, Pululu P, et al. [Sociodemographic, biological and clinical profile of patients living with HIV during screening in a voluntary counselling and screening centre in a rural area of Mbanza-Ngungu, Democratic Republic of Congo, in 2006-2011]. Bull Soc Pathol Exot 2013;106:180-3.

[14] Nthumba PM. Palliative reconstructive surgery: contextualizing palliation in resource-poor settings. Plast Surg Int 2014;2014:275215.

[15] Wiese CH, Lassen Cl, Bartels UE, et al. International recommendations for outpatient palliative care and prehospital palliative emergencies - a prospective questionnaire-based investigation. BMC Palliat Care 2013;12:10.

[16] Krzesinski JM, Sumaili KE, Cohen E. How to tackle the avalanche of chronic kidney disease in sub-saharan Africa: the situation in the Democratic Republic of Congo as an example. Nephrol Dial Transplant 2007;22:332-5.
[17] Oni T, Youngblood E, Boulie A, McGrath N, Wilkinson RJ, Levitt NS. Patterns of HIV, TB, and non-communicable disease multimorbidity in peri-urban South Africa - a cross sectional study. BMC Infect Dis 2015;15:20.

[18] Coresh J, Selvin E, Stevens LA, et al. Prevalence of chronic kidney disease in the United States. JAMA 2007;298:2038-47.

[19] Otero A, Gayoso P, Garcia F, de Francisco AL. Epidemiology of chronic renal disease in the Galician population: results of the pilot Spanish EPIRCE study. Kidney Int 2005;99:S16-9.

[20] Ninomiya T, Kiyohara Y, Kubo M, et al. Chronic kidney disease and cardiovascular disease in a general Japanese population: the Hisayama Study. Kidney Int 2005;68:228-36.

[21] Clark WF, Macnab JJ, Chen SJ, Suri R, Moist L, Garg AX. Evaluation of GFR estimating equations in the general community: implications for screening. Clin J Am Soc Nephrol 2006;1:787-95

[22] Cirillo M, Laurenzi M, Mancini M, Zanchetti A, Lombardi C, De Santo NG. Low glomerular filtration in the population: prevalence, associated disorders, and awareness. Kidney Int 2006;70:800-6.

[23] Hallan SI, Cresh J, Astor BC, et al. International comparison of the relationship of chronic kidney disease prevalence and ESRD risk. J Am Soc Nephrol 2006;17:2275-84.

[24] Sumaili EK, Krzesinski JM, Cohen EP, Sseka NM. [Epidemiology of chronic kidney disease in the Democratic Republic of Congo: review of cross-sectional studies from Kinshasa, the capital]. Nephrol Ther 2010;6:232-9.

[25] UNDESA. Population ageing and non-communicable diseases; 2012 [http://www.un.org/en/development/desa/ population/publications/pdf/popfacts/popfacts_2012-1.1.pdf Consulté le 16/02/2015].

[26] Organisation mondiale de la santé. Les maladies non transmissibles; 2013 [http:/www.who.int/features/factlles/ noncommunicable.disease/fr consulté le 05/10/2014].

[27] Holmes MD, Dalai S, Volmink J, et al. Non-communicable diseases in sub-Saharan Africa: the case for cohort studies. PLoS Med 2010;7:e1000244.

[28] Organisation mondiale de la santé. Cancer; 2015 [http://www.who.int/mediacentre/factsheets/fs297/fr/ consulté le 20/12/2015].

[29] Yach D, Hawkes C, Goud CL, Hofman KJ. The global burden of chronic diseases: overcoming impediments to prevention and control. JAMA 2004;291:2616-22.

[30] Katchunga PB, M’buyamba-kayamba JR, Masumbuku BE, et al. [Hypertension in the adult Congolese population of Southern Kivu: results of the Vitaraa study]. Presse Med 2011;40:e315-23.

[31] Makulo RJ, Nseka MN, Jadoul M, et al. [Albuminuria during the screening for diabetes in a semi-rural area (Kisantu City, DR Congo)]. Nephrol Ther 2010;6:513-9.

[32] Ekulu PM, Nseka NM, Aloni MN, et al. [Prevalence of proteinuria and its association with HIV/AIDS in Congolese children living in Kinshasa, Democratic Republic of Congo]. Nephrol Ther 2012;8:163-7.

[33] Longo-Mbenza B, Ngoma DV, Nahimana D, et al. Screen detection and the WHO stepwise approach to the prevalence and risk factors of arterial hypertension in Kinshasa. Eur J Cardiovasc Prev Rehabil 2008;15:503-8.

[34] Mvitu Muaka M, Longo-Mbenza B. Causes of visual disability among Central Africans with diabetes mellitus. Afr Health Sci 2012;12:193-7.

[35] Ngoie Maloba V, Chenge Borasisi G, Kaimbo wa Kaimbo D, Snyers B. [Diabetic retinopathy in Lubumbashi]. Bull Soc Belge Ophtalmol 2012;319:51-9.

[36] Pakasa NM, Sumaili KE. [Pathological peculiarities of chronic kidney disease in patient from sub-Saharan Africa. Review of data from the Democratic Republic of the Congo]. Ann Pathol 2012:32:40-52. 
[37] Programme des Nations unies pour le développement. Province de Kinshasa: profil résumé. In: Pauvreté et conditions de vie des ménages. Kinshasa: Unité de lutte contre la pauvreté; 2009.

[38] Ministère du plan RDC. Deuxième enquête démographique et de santé (EDS-RDC II 2013-2014); 2014 [https://dhsprogram.com/pubs/pdf/FR300/FR300.pdf consulté le 28/05/2016].

[39] Organisation mondiale de la santé. Combatre le VIH/SIDA, tuberculose, paludisme et autres maladies; 2014 [www.aho.afro.int/profiles_information/index.php/ democratic_republic_of_congo: MDG_6: combat_HIV/AIDS_TB, malaria_and_other_disease/fr consulté le 28/05/2016].

[40] Oates KR. A survey of malignant disease in Zaire. Fam Pract $1986 ; 3: 102-6$.

[41] Budiongo AN, Ngiyulu RM, Lebwaze BM, et al. Pediatric nonHodgkin lymphomas: first report from Central Africa. Pediatr Hematol Oncol 2015;32:239-49.

[42] Wakamb GK, Nkashama GM, Mbuli RL, Borasisi GC, Nikulu Jl. [Problematic of the management of childhood cancer: experience of retinoblastoma in Lubumbashi (DR Congo) and the importance of early diagnosis]. Pan Afr Med J 2013; 14:64.

[43] Organisation mondiale de la santé. WHO guidelines on persisting pain in children; 2012 [http://www.who.int/ medicines/areas/quality_safety/guide_perspainchild/en/ consulté ce 12/12/2016].

[44] Economist T. Growing pains: poor countries are developing the diseases of the rich, with lethal consequences; 2011 [http://www.economist.com/node/21530099 consulté le $16 / 02 / 2015]$.

[45] Draper CA, Draper CE, Bresick GF. Alignment between chronic disease policy and practice: case study at a primary care facility. PLoS One 2014;9:e105360.

[46] Sci Dev Net. Maladies chroniques: faits et chiffres; 2008 [http://www.scidev.net/afrique-sub-saharienne/sante/ article-de-fond/maladies-chroniques-faits-et-chiffres.html consulté le 03/11/2014].

[47] Rachel N. Where have all the donors gone? Scarce donor funding for non-communicable diseases; 2011 [http://www.cgdev.org/sites/default/files/1424546_file_ Nugent_Feigl_NCD_FINAL.pdf consulté le 16/02/2015].

[48] Hartwig K, Dean M, Hartwig K, Mmbando PZ, Sayed A, de Vries $\mathrm{E}$. Where there is no morphine: the challenge and hope of palliative care delivery in Tanzania. Afr J Prim Health Care Fam Med 2014;6:E1-8.
[49] Organisation mondiale de la santé. Les soins palliatifs; 2015 [http://www.who.int/cancer/palliative/fr/ consulté le 06/07/2015].

[50] Downing J, Knapp C, Muckaden MA, Fowler-Kerry S, Marston J, ICPCN Scientific committee. Priorities for global research into children's palliative care: results of an international Delphi study. BMC Palliat Car 2015;14:36.

[51] Lofandjola Masumbuku J, Coppieters Y. [Qualitative analysis of palliative care and support in medical practices in DRC]. Med Sante Trop 2014;24:83-8.

[52] Department of economic social affairs. World urbanization prospects: the 2003 revision. New York: United Nations; 2004. p. 2004 [Consulté le 02/02/2015].

[53] Manzambi J, Bertrand F, Albert A, Reginster JY, Van Balen H. Les déterminants du comportement de recours au centre de santé en milieu urbain africain : résultats d'une enquête de ménage menée à Kinshasa, Congo. Trop Med Int Health 2000;5:563-70.

[54] Graham N, Gwyther L, Tiso T, Harding R. Traditional healers' views of the required processes for a "good death" among Xhosa patients pre- and post-death. J Pain Symptom Manage 2013;46:386-94.

[55] Office fédéral des migrations O.D.M. Focus RD Congo : Le système sanitaire à Kinshasa : médicaments et soins du VIHsida, de l'hypertension artérielle, du diabète de type II et des troubles mentaux; 2014 [https://www.sem.admin.ch/dam/ data/sem/internationales/herkunftslaender/afrika/cod/CODmed-lage-f.pdf consulté le 12/05/2017].

[56] Service Public Fédéral. Sécurité de la chaine alimentaire et environnement. In: Statistiques annuelles des professionnels des soins de santé en Belgique; 2012.

[57] Kikule E. A good death in Uganda: survey of needs for palliative care for terminally ill people in urban areas. BMJ 2003;327:192-4.

[58] Gysels M, Pell C, Straus L, Pool R. End of life care in sub-Saharan Africa: a systematic review of the qualitative literature. BMC Palliat Care 2011;10:6.

[59] Young T., Busgeeth K., Home-based care for reducing morbidity and mortality in people infected with HIV/AIDS. Cochrane Database Syst Rev:17;0054.

[60] Ministère de la santé RDC. Stratégie de renforcement du système de santé; 2006 [www.nyankunde.org/documentation/ SRSS\%2OVERSION\%20FINALE.pdf consulté le 16/02/2015].

[61] Ministère de la santé RDC. Liste nationale des médicaments essentiels; 2010 [http://apps.who.int/medicinedocs/ documents/s18817fr/s18817fr.pdf consulté le 16/02/2015]. 